\title{
Analysis of Therapeutic Targets and Prognostic Biomarkers Among semaphorins in the glioblastoma Microenvironment
}

\section{Fan Jiang}

The First Affiliated Hospital of Soochow University

\section{Yanbo Yang}

China-Japan Friendship Hospital

\section{Zilan Wang}

The First Affiliated Hospital of Soochow University

\section{Guangjie Liu}

The First Affiliated Hospital of Soochow University

\section{Xiaohong Du}

The First Affiliated Hospital of Soochow University

\section{Zhong Wang ( $\sim$ wangzhong761@163.com )}

The First Affiliated Hospital of Soochow University

\section{Research}

Keywords: glioblastoma, bioinformatics analysis, tumor microenvironment, biomarker, SEMA

Posted Date: September 21st, 2021

DOl: https://doi.org/10.21203/rs.3.rs-903382/v1

License: (1) This work is licensed under a Creative Commons Attribution 4.0 International License. Read Full License 


\section{Abstract \\ Background}

Glioblastoma (GBM) is the most invasive and common form among brain cancers in adults. GBM is characterized for its poor survival and markedly high tumors heterogeneity with shortage of effective therapies. Semaphorins, a family of membrane-associated and secreted proteins, were originally defined as neuronal growth pyramidal proteins involved in directing repulsive axons. Semaphorins are repeatedly involved in the evolution of neural circuits, involving not only the generation and guidance of neurons, but also the recognition of target regions and cells and synapse formation. However, the differential expression and clinical prognostic value of semaphorins in GBM were not yet available clarity.

\section{Methods}

In this study, various databases including ONCOMINE (1159 samples), GEPIA (TCGA and GTEx dataset), UALCAN (samples from TCGA dataset), cBioPortal (604 samples from GDAC firehose of TCGA dataset), GeneMANIA(data from publicly available daatbases, e.g. GEO, BioGRID) and TIMER(samples from TCGA) were exploited.

\section{Results}

We found that in GBM tissues the transcriptional levels of SEMA3A/3B/3E/3F/5A/6A were markedly elevated, while those of $S E M A 3 G / 4 A / 4 D / 4 F / 5 B$ were markedly reduced. GBM patients with lower levels of SEMA3F/4F transcription had a significantly better outcome. The function of semaphorins was mainly related to the regulation of cell growth and development. Besides, we found that the expression of semaphorin was significantly correlated with the infiltration of immune cells.

\section{Conclusions}

Semaphroins are differentially expressed in glioblastoma compared with normal brain tissue. They could provide diverse prognostic values, participate in various molecular pathways. In addition, the semaphorin protein family plays an important role in immune cell infiltration. Our study may supply potential target genes for the treatment of glioblastoma while providing new insights into the selection of prognostic biomarkers. Besides, our results may offer new insights into immunotherapy targeting and analysis of protein co-expression in SEMA for glioblastoma.

\section{Introduction}

Glioblastoma (GBM) is a primary brain tumor with poor survival (i.e., overall median survival time is one year[1]). It is characterized for its markedly high tumor heterogeneity[2], and lacking effective therapies 
[3]. The standard treatment currently in use consists of maximal surgical resection of the tumor, which is followed by precision radiotherapy with adjacent chemotherapy. With the increasing availability of highthroughput genomic, genetic, and epigenetic data, bioinformatics analysis provides a full range of molecular mechanisms for the formation and development of GBM. However, with such complex intratumoral molecular heterogeneity, additional therapeutic targets and biomarkers of prognosis must be identified.

Semaphorins (SEMA), originally identified as neuronal growth cone-fold proteins involved in directing repulsive axons, are a family of membrane-associated and secreted proteins [4-11]. Semaphorins are involved in the entire evolution of neural circuits, including the growth and guidance of neurons, the identification of target regions and cells, and synapse formation.

Although the adjustment of semaphorin receptors remains incompletely understood, the intricate molecular mechanisms that diversify semaphorin responsiveness and spatiotemporal control have attracted more attention. The rapid development of next-generation gene sequencing technologies and the establishment of multiple oncogene databases have made it possible to analyze semaphorins at the transcriptional and expression levels. In this study, we conducted a bioinformatics analysis of semaphorins expression in GBM based on several large public databases. Besides, we evaluated their potential to serve as therapeutic targets and prognostic biomarkers to provide more information for the management of GBM.

\section{Materials And Methods}

\section{ONCOMINE}

ONCOMINE provides powerful bioinformatics service at translational level for whole genome expression analysis [12] This study retrieved data to estimate the expression of semaphorins in GBM with a p-value of 0.05 and a fold change of 2 , with a gene ranking in the top $10 \%$ as the threshold of significance. The differences in the expression of semaphorins in GBM were analyzed using Student's t-test.

\section{UALCAN}

UALCAN provides analytical tools based on data retrieved from the Cancer Genome Atlas (TCGA) [13]. Expressional level data of semaphorins were acquired by using UALCAN's "Expression Analysis" module and the "GBM" dataset. The P-value cut-off point was 0.05 in this study.

\section{GEPIA}

GEPIA is a bioinformatics analysis platform developed by Peking University containing RNA sequence expression data from more than 9000 tumor samples and over 8000 normal tissue samples[14]. This study utilized the "Single Gene Analysis" module of GEPIA to perform differential expression analysis of mRNA in glioblastoma and normal tissues and prognostic analysis associated with semaphorins. Besides, we conducted a multi-gene comparative analysis of semaphorins with the "GBM" dataset in 
"Multiple Gene Comparison" module of GEPIA. All these analyses were conducted with the Student's t-test and $p$-values for expression analysis were cut-off value of 0.05 .

\section{cBioPortal}

The cBioPortal is a website for visualizing and analyzing cancer genomics data from multi-dimensional angles[15]. Network modules for gene alterations and co-expression were obtained from cBioPortal based on the TCGA database. 604 glioblastoma samples (TCGA) were analyzed. A z-score threshold of \pm 2.0 was used to obtain mRNA expression z-scores (RNA Seq V2 RSEM). We set the z-score cutoff to \pm 2.0 and get the line expressing the z-scores.

\section{GeneMANIA}

GeneMANIA, a web-based bioinformatical tool-kit, provides information on gene and protein coexpression, interactions, pathways, and domain similarities of genes submitted[16].

\section{TIMER}

TIMER is an editable visualization tool for the systematic assessment of different immune cell infiltrates and their corresponding clinical significance[17]. In this study, the "gene module" was utilized to assess the relationship between semaphorins levels and infiltration of immune cells. We used the 'survival module' to assess the relationship between clinical outcomes and immune infiltration with expression of smepahorins.

\section{Results}

\section{Aberrant Expression of semaphorins in Patients with GBM}

We retrieved twenty semaphorins (SEMA3A, SEMA3B, SEMA3C, SEMA3D, SEMA3E, SEMA3F, SEMA3G, SEMA4A, SEMA4B, S3MA4C, SEMA4D, SEMA4F, SEMA4G, SEMA5A, SEMA5B, SEMA6A, SEMA6B, SEMA6C, SEMA6D, SEMA7A) in ONCOMINE. Firstly, the differences of semaphorins at transcriptional levels in GBM vs. normal brain tissues in ONCOMINE were explored. We can see the preliminary results from Figure 1 and Table 1. There was a significant increase in the transcription levels of SEMA3A, SEMA3B, SEMA3E, SEMA3F, SEMA5A, and SEMA6A in GBM vs. normal tissues and a significant decrease in the transcription levels of SEMA3G, SEMA4A, SEMA4D, SEMA4F, and SEMA5B in GBM and normal brain tissues. The data is in accordance with previous studies. Lee et al. revealed a significant upregulation of SEMA3A in the glioblastoma microenvironment[18]. Liang et al. found SEMA3B ( $p$-value $=5.89 \mathrm{E}-5$ ) in GBM was significantly elevated with a fold change of 13.262[19] at transcriptional level. Another similar study conducted by Lee et al. also found the level of SEMA3E and SEMA3F was increased in GBM[18].

The transcriptional levels of SEMA3G in GBM were lower than these in normal brain tissues in the TCGA dataset (Table 1). The results of Bredel et al. [20]and the TCGA dataset suggested that SEMA4A 
expression in GBM vs. that in normal brain tissues was fallen. SEMA4D[21]in GBM were remarkably lower at transcriptional levels in the results reported by Murat et al. [21](fold change $=-5.566$ and $p$-value $=$ 0.005). The fold change of SEMA4F was -2.690 ( $p$-value $=0.001)$ in the dataset of TCGA and -2.188 ( $p$ value $=0.003$ ) in the dataset of Bredel et al[20]. In the dataset of TCGA and Murat et al.[21], the transcriptional levels of SEMA5A in GBM were higher than these in normal brain tissues, while the transcriptional levels of SEMA5B in GBM were lower than these in normal tissues in the dataset of Lee et al.[18]. Moreover, significantly increased levels of SEMA6A were found in GBM[21]. We also verified the difference in expression levels of semaphorins in GBM and normal tissues using UALCAN. SEMA3A $(p=3.90 e-03)$, SEMA3F ( $p=5.90 e-5)$, SEMA4B $(p=7.66 e-3)$, and SEMA4G $(p=4.65 e-2)$ had significantly higher transcriptional levels, while SEMA4A ( $p=3.78 \mathrm{e}-2)$ had reduced transcriptional levels as expected (Figure 2). Then we made a comparison of the levels of relative expression of semaphorins in GBM. We found SEMA6A had the highest relative expression of all the semaphorins we evaluated. (Figure3). To further identify the association between semaphorins and GBM progression and clinical outcomes, we evaluated all semaphorins that were differentially expressed in GBM versus normal tissues. (SEMA3A, SEMA3B, SEMA3E, SEMA3F, SEMA3G, SEMA4A, SEMA4D, SEMA4F, SEMA5A, SEMA5B, SEMA6A,). We excluded SEMA3C, SEMA3D, SEMA4B, SEMA4C, SEMA4G, SEMA6C, SEMA6B, SEMA7A, and SEMA6D from further analysis because their expression differences in GBM and normal tissues were not significant enough.

\section{The Prognostic Value of semaphorins in Patients with GBM}

We evaluated the correlation between differentially expressed semaphorins and clinical outcomes with GEPIA to assess the value of differentially expressed semaphorins in GBM progression. And we can see all these disease-free survival outcomes in Figure 4. Patients with low transcriptional levels seemed to have longer disease-free survival (SEMA4F $(p=7.4 \mathrm{E}-4)$ ). We also evaluated the value of different semaphorins in GBM patients about the overall survival. It was found that GBM clinical cases with low transcriotion in SEMA3F $(p=0.0013)$ and SEMA4F $(p=0.0073)$ were significantly associated with longer overall survival (Figure 5).

\section{SEMA Family Gene Alteration, Co-Expression, Neighbor Gene Network, and Interaction Analysis in GBM Patients}

A comprehensive molecular characterization of the different semaphorins at expressional levels was conducted. A temporary dataset from TCGA was used to profile genetic alterations in differentially expressed semaphorins. We found that SEMA3A(3\%), SEMA3B(1.2\%), SEMA3D,(2.8\%), SEMA3E(3\%), SEMA3F(2.1\%), SEMA3G,(1.9\%), SEMA4A(2.3\%), SEMA4B(1.2\%), SEMA4D(2.1\%), SEMA4F(2.4\%). SEMA4G(2.1\%), SEMA5A(2.8\%), SEMA5B(2.6\%), SEMA6A(1.4\%), SEMA6B(2.4\%), and SEMA7A(1.9\%) were altered in the queried GBM samples, respectively (Figure 6). Results from GeneMANIA also revealed that the differential expressed semaphorins (SEMA3A, SEMA3B, SEMA3C, SEMA3D, SEMA3E, SEMA3F, SEMA3G, SEMA4A, SEMA4B, SEMA4D, SEMA4F, SEMA4G, SEMA5A, SEMA5B, SEMA6A, SEMA6B and 
SEMA7A) were primarily related to regulation of cell morphogenesis, develpmental cell growth and regulation of extent of cell growth (Figure 7).

\section{Immune Cell Infiltration of semaphorins in GBM Patients}

Semaphorins have been demonstrated to participate in the inflammatory response and infiltration of immune cells, thereby influencing clinical outcomes in patients with GBM. In this way, we began to investigate the relationship between different genes at expressional levels. And we conducted this work with TIMER database. We found that SEMA3B was positively correlated with the infiltration of CD4+ T cells (Cor $=0.205, p=2.43 e-5$ ) at expressional levels. And we found a negative association between SEMA3E and the infiltration of $B$ cells (Cor $=-0.12, p=1.41 e-2)$ and positive realationship between SEMA3E expression and the infiltration of macrophage (Cor $=0.132, p=6.87 \mathrm{e}-3)$. There was a negative association between SEMA3F expression and the infiltration of $B$ cells (Cor $=-0.159, p=1.09 \mathrm{e}-3$ ), CD8+ T cells (Cor $=-0.108, p=2.70 e-02$ ) and neutrophils (Cor $=-0.11, p=2.41 e-02)$. Accordingly, there was a positive correlation between the expression of SEMA3G and the infiltration of $B$ cells (Cor $=0.13, p=$ $7.90 \mathrm{e}-03)$. We found that SEMA4A expression was negatively associated with the infiltration of CD8+ T cells (Cor $=-0.167, p=5.85 \mathrm{e}-4$ ) and positively correlated with infiltration of CD4+T cells (Cor $=0.096, p=$ 4.96e-2), neutrophils (Cor $=0.144, p=3.26 \mathrm{e}-03$ ) and dendritic cells (Cor $=0.111, p=2.30 \mathrm{e}-2$ ). It is found that the CD8+T cells is lower in immune infiltration, while SEMA4D is relatively higher (Cor $=-0.168, p=$ $5.71 \mathrm{e}-4$ ) at expressional levels. We found that SEMA4F expression was negatively associated with the infiltration of $B$ cells (Cor $=-0.17, p=4.66 \mathrm{e}-4$ ) and positively associated with the dendritic cells (Cor $=$ $0.11, p=2.40 \mathrm{e}-2)$. We also found a positive correlation between SEMA5A expression and CD8+ T cells (Cor $=0.24, p=8.82 \mathrm{e}-4$ ) and CD $4+T$ Cells (Cor $=0.157, p=1.323-3$ ) infiltration. SEMA5B expression is negatively related with the infiltration of $B$ cells (Cor $=-0.201, p=2.37 e-2)$ while SEMA5B expression is positively associated with the macrophages (Cor=-0.176, $p=4.48 \mathrm{e}-2$ ) in infiltration. Moreover, SEMA6A expression was found to have a positive correlation between infiltration of all immune cell types except for $B$ cells and dendritic cells. All the related figures were plotted in the supplement.

\section{Discussion}

We first explored the expression of semaphorins. 11 genes were found to differentially expressed in GBM compared to in normal tissues (upregulation of SEMA3A, SEMA3B, SEMA3E, SEMA3F, SEMA5A, and SEMA6A; downregulation of SEMA3G, SEMA4A, SEMA4D, SEMA4F, and SEMA5B). Patients with GBM with low expression of SEMA3F and SEMA4F were significantly related to better overall survival, and patients with low expression of SEMA4F had a significant relationship to both better disease-free survival and overall survival. These data suggest that different semaphorins at expressional levels could play an important part in GBM. Datasets from Murat[21] and TCGA suggested that SEMA6A expression is significantly enhanced in brain tissues. Furthermore, we found that SEMA3A, SEMA4D, SEMA5A and SEMA7A take a significant part cell morphogenesis about regulation, growth of develpmental cells and regulation of extent of cell growth from GeneMANIA. 
It is increasingly clear that semaphorins influence various cellular processes in the development of the nervous system, from neuronal migration[22] and axonal trimming[23, 24] to synapse formation and function[25]. Many effects are dependent on their ability to act as repulsive or attractive proteins that can control the cytoskeleton[26,27]. There is growing evidence that various semaphorins are involved in almost all phases of the physiological and pathological immune response[28]. Semaphorins consists of a vast family of conserved proteins that are further subdivided into different types of proteins based on their structure and sequence similarity $[1,29]$. The signal of semaphorins is primarily mediated by Plexin and Neuropilin receptors[30]. Besides, recent studies show that the development of neurons can be influenced by semaphorins through downstream targets. Although semaphorins are most well-known for their role in the development and growth of neurons, their involvement in malignant tumors is receiving increasing attention. While reviews of semaphorins in cancer[29, 31,32] and semaphorins as therapeutic targets[33] are available and detailed elsewhere, the prognostic value and biological function of the SEMA family have not been well characterized. Previous studies lacked attention to SEMA3F and SEMA4F, yet we have found the prognostic value of these two proteins in GBM in this study, which we did not anticipate.

We explored the molecular characteristics of several semaphrins in GBM for some semaphorins are significantly differentially expressed in GBM. Differentially expressed semaphorins are often genetically altered in GBM. Glioblastoma tumorigenesis and progression is a complex, multifaceted process in which genetic alterations play an important role[34]. Diverse correlations between different semaphorins at expressional levels suggested that some members of semaphorins take a synergistic part in the tumorigenesis and progression of GBM.

Previous researches have paid much attention to semaphorins in the nervous system, the circulatory system, and the immune system[35]. A growing body of evidence suggests that the infiltration of immune cells may impact tumor progression and recurrence. Besides, the infiltration of immune cells could serve as a significant determining factor in response to clinical outcomes[36, 37]. CD 4 + T cells recognize cancer antigens, and activating M1 macrophages can suppress cancer growth[38]. SEMA family expression was remarkably correlated with the infiltration of six major types of immune cells: $B$ cells, CD8 + T cells, CD 4 + T cells, macrophages, neutrophils, and dendritic cells, indicating that CXC chemokine can be used as a prognostic indicator and a reflection of status in immune system.

This study has some limitations. The analysis of molecular interactions could be performed more intensively and elaborately. In addition, another independent cohort could be performed to verify our results.

\section{Conclusion}

We analyzed the differences of transcriptional and expressional levels, prognostic values, and immune cell infiltration of all of the SEMA family members using the Oncomine database, UCLAN, GEPIA, cBioPortal, GeneMAINA and TIMER. Among all the semaphorins available on these databases, we 
revealed that the mRNA expression levels of SEMA3A, SEMA3B, SEMA3E, SEMA3F, SEMA5A, and SEMA6A were upregulated; while SEMA3G, SEMA4A, SEMA4D, SEMA4F, and SEMA5B were downregulated in the GBM microenvironment. Further analysis indicated that patients with low expression of SEMA3F and SEMA4F were related to better overall survival and patients with low expression of SEMA4F were significantly related to better disease-free survival, suggesting that the two genes may be potential targets in the future chemotherapy.

Eleven SEMA members were significantly correlated with GBM patients. These eleven SEMA proteins especially SEMA3A, SEMA4D, SEMA5A and SEMA7A take an important part in the regulation of cell morphogenesis, developmental growth and regulation of extent of cell growth. Besides, we found that SEMA family expression was remarkably related to the infiltration. More sophisticated mechanism studies and large-data clinical trials remain to be implemented to explore the more detailed function of the SEMA family in GBM. Every SEMA member might show its functions through different kinds of signal pathways.

$\mathrm{n}$ future research, we can pay more attention to the transcription, expression and prognosis of SEMA3F and SEMA4F and GBM. In conclusion, our study provides new insights to precisely design new immunotherapies to help clinicians select appropriate targeted agents and prognostic biomarkers for GBM patients to more accurately select cost-effective therapies.

\section{List Of Abbreviations}

SEMA, semaphoring; GBM, glioblastoma;

\section{Declarations}

\section{AUTHOR CONTRIBUTIONS}

ZW and XHD: conceptualization. YBY and ZLW: data curation. FJ: formal analysis. FJ, YBY, and ZLW: methodology. FJ and YBY: project administration. XHD: visualization. FJ, YBY and ZLW: writing - original draft. And all authors contributed to the article and approved the submitted version.

\section{Ethics approval and consent to participate}

All procedures performed in studies involving human participants were in accordance with the ethical standards of the institutional and/or national research committee and with the 1964 Helsinki Declaration and its later amendments or comparable ethical standards. The study was approved by the Institutional Review Board of the First Affiliated hospital of Soochow University (No. 2019117). As this article is a retrospective study, ethics committees have been granted exemption of patient informed consent.

\section{Consent for publication}

Not applicable. 
Availability of data and materials

The datasets generated during and/or analyzed during the current study are available in the ONCOMINE, TCGA, Cbioportal dataset.

Competing interests

The authors declare that they have no Competing interests.

\section{Funding}

This work was supported by the Suzhou Health Talents Training Project (GSWS2019002).

\section{AUTHOR CONTRIBUTIONS}

ZW and XHD: conceptualization. YBY and ZLW: data curation. FJ: formal analysis. FJ, YBY, and ZLW: methodology. FJ and YBY: project administration. XHD: visualization. FJ, YBY and ZLW: writing - original draft. And all authors contributed to the article and approved the submitted version.

\section{Acknowledgments}

Not applicable.

\section{References}

1. Unified nomenclature for the semaphorins/collapsins. Semaphorin Nomenclature Committee. Cel/1999, 97(5):551-552.

2. Kolodkin AL, Tessier-Lavigne M: Mechanisms and molecules of neuronal wiring: a primer. Cold Spring Harb Perspect Bio/2011, 3(6).

3. Koncina $E$, Roth $L$, Gonthier B, Bagnard D: Role of semaphorins during axon growth and guidance. Adv Exp Med Bio/2007, 621:50-64.

4. Kumanogoh A, Kikutani $\mathrm{H}$ : Semaphorins and their receptors: novel features of neural guidance molecules. Proc Jpn Acad Ser B Phys Biol Sci2010, 86(6):611-620.

5. Mann F, Chauvet S, Rougon G: Semaphorins in development and adult brain: Implication for neurological diseases. Prog Neurobio/2007, 82(2):57-79.

6. Ohgaki $\mathrm{H}$, Kleihues $\mathrm{P}$ : Population-based studies on incidence, survival rates, and genetic alterations in astrocytic and oligodendroglial gliomas. J Neuropathol Exp Neuro/2005, 64(6):479-489.

7. Pasterkamp RJ, Giger RJ: Semaphorin function in neural plasticity and disease. Curr Opin Neurobio/2009, 19(3):263-274. 
8. Patel AP, Tirosh I, Trombetta JJ, Shalek AK, Gillespie SM, Wakimoto H, Cahill DP, Nahed BV, Curry WT, Martuza RLet al: Single-cell RNA-seq highlights intratumoral heterogeneity in primary glioblastoma. Science2014, 344(6190):1396-1401.

9. Tran TS, Kolodkin AL, Bharadwaj R: Semaphorin regulation of cellular morphology. Annu Rev Cell Dev Bio/2007, 23:263-292.

10. Zong H, Verhaak RG, Canoll P: The cellular origin for malignant glioma and prospects for clinical advancements. Expert Rev Mol Diagn2012, 12(4):383-394.

11. Yazdani U, Terman JR: The semaphorins. Genome Bio/2006, 7(3):211.

12. Rhodes DR, Yu J, Shanker K, Deshpande N, Varambally R, Ghosh D, Barrette T, Pandey A, Chinnaiyan AM: ONCOMINE: a cancer microarray database and integrated data-mining platform. Neoplasia2004, 6(1):1-6.

13. Chandrashekar DS, Bashel B, Balasubramanya SAH, Creighton CJ, Ponce-Rodriguez I, Chakravarthi B, Varambally S: UALCAN: A Portal for Facilitating Tumor Subgroup Gene Expression and Survival Analyses. Neoplasia2017, 19(8):649-658.

14. Tang Z, Li C, Kang B, Gao G, Li C, Zhang Z: GEPIA: a web server for cancer and normal gene expression profiling and interactive analyses. Nucleic Acids Res2017, 45(W1):W98-W102.

15. Gao J, Aksoy BA, Dogrusoz U, Dresdner G, Gross B, Sumer SO, Sun Y, Jacobsen A, Sinha R, Larsson Eet al: Integrative analysis of complex cancer genomics and clinical profiles using the cBioPortal. Sci Signal2013, 6(269):pl1.

16. Warde-Farley D, Donaldson SL, Comes O, Zuberi K, Badrawi R, Chao P, Franz M, Grouios C, Kazi F, Lopes CT et al: The GeneMANIA prediction server: biological network integration for gene prioritization and predicting gene function. Nucleic Acids Res2010, 38(Web Server issue):W214-220.

17. Li T, Fan J, Wang B, Traugh N, Chen Q, Liu JS, Li B, Liu XS: TIMER: A Web Server for Comprehensive Analysis of Tumor-Infiltrating Immune Cells. Cancer Res2017, 77(21):e108-e110.

18. Zeng Q, Sun S, Li Y, Li X, Li Z, Liang H: Identification of Therapeutic Targets and Prognostic Biomarkers Among CXC Chemokines in the Renal Cell Carcinoma Microenvironment. Front Onco/2019, 9:1555.

19. Liang Y, Diehn M, Watson N, Bollen AW, Aldape KD, Nicholas MK, Lamborn KR, Berger MS, Botstein D, Brown POet al: Gene expression profiling reveals molecularly and clinically distinct subtypes of glioblastoma multiforme. Proc Natl Acad Sci U S A2005, 102(16):5814-5819.

20. Bredel M, Bredel C, Juric D, Harsh GR, Vogel H, Recht LD, Sikic BI: Functional network analysis reveals extended gliomagenesis pathway maps and three novel MYC-interacting genes in human gliomas. 
Cancer Res2005, 65(19):8679-8689.

21. Murat A, Migliavacca E, Gorlia T, Lambiv WL, Shay T, Hamou MF, de Tribolet N, Regli L, Wick W, Kouwenhoven MCet al: Stem cell-related "self-renewal" signature and high epidermal growth factor receptor expression associated with resistance to concomitant chemoradiotherapy in glioblastoma. J Clin Onco/2008, 26(18):3015-3024.

22. Hernandez-Miranda LR, Cariboni A, Faux C, Ruhrberg C, Cho JH, Cloutier JF, Eickholt BJ, Parnavelas JG, Andrews WD: Robo1 regulates semaphorin signaling to guide the migration of cortical interneurons through the ventral forebrain. J Neurosci2011, 31(16):6174-6187.

23. Bagri A, Cheng HJ, Yaron A, Pleasure SJ, Tessier-Lavigne M: Stereotyped pruning of long hippocampal axon branches triggered by retraction inducers of the semaphorin family. Cel/2003, 113(3):285-299.

24. Low LK, Liu XB, Faulkner RL, Coble J, Cheng HJ: Plexin signaling selectively regulates the stereotyped pruning of corticospinal axons from visual cortex. Proc Natl Acad Sci U S A2008, 105(23):8136-8141.

25. Carrillo RA, Olsen DP, Yoon KS, Keshishian H: Presynaptic activity and CaMKII modulate retrograde semaphorin signaling and synaptic refinement. Neuron2010, 68(1):32-44.

26. Schwamborn JC, Fiore R, Bagnard D, Kappler J, Kaltschmidt C, Puschel AW: Semaphorin 3A stimulates neurite extension and regulates gene expression in PC12 cells. J Biol Chem2004, 279(30):30923-30926.

27. Toyofuku T, Nojima S, Ishikawa T, Takamatsu H, Tsujimura T, Uemura A, Matsuda J, Seki T, Kumanogoh A: Endosomal sorting by Semaphorin 4A in retinal pigment epithelium supports photoreceptor survival. Genes Dev2012, 26(8):816-829.

28. Vadasz Z, Toubi E: Semaphorins: their dual role in regulating immune-mediated diseases. Clin Rev Allergy Immunol2014, 47(1):17-25.

29. Neufeld G, Shraga-Heled N, Lange T, Guttmann-Raviv N, Herzog Y, Kessler O: Semaphorins in cancer. Front Biosci2005, 10:751-760.

30. Tamagnone L, Artigiani S, Chen H, He Z, Ming GI, Song H, Chedotal A, Winberg ML, Goodman CS, Poo Met al: Plexins are a large family of receptors for transmembrane, secreted, and GPI-anchored semaphorins in vertebrates. Ce//1999, 99(1):71-80.

31. Rehman M, Tamagnone L: Semaphorins in cancer: biological mechanisms and therapeutic approaches. Semin Cell Dev Bio/2013, 24(3):179-189.

32. Capparuccia L, Tamagnone L: Semaphorin signaling in cancer cells and in cells of the tumor microenvironment-two sides of a coin. J Cell Sci2009, 122(Pt 11):1723-1736. 
33. Worzfeld T, Offermanns S: Semaphorins and plexins as therapeutic targets. Nat Rev Drug Discov2014, 13(8):603-621.

34. Yap NY, Rajandram R, Ng KL, Pailoor J, Fadzli A, Gobe GC: Genetic and Chromosomal Aberrations and Their Clinical Significance in Renal Neoplasms. Biomed Res Int2015, 2015:476508.

35. Alto LT, Terman JR: Semaphorins and their Signaling Mechanisms. Methods Mol Bio/2017, 1493:1-25.

36. Bindea G, Mlecnik B, Tosolini M, Kirilovsky A, Waldner M, Obenauf AC, Angell H, Fredriksen T, Lafontaine L, Berger Aet al: Spatiotemporal dynamics of intratumoral immune cells reveal the immune landscape in human cancer. Immunity2013, 39(4):782-795.

37. Liu X, Wu S, Yang Y, Zhao M, Zhu G, Hou Z: The prognostic landscape of tumor-infiltrating immune cell and immunomodulators in lung cancer. Biomed Pharmacother2017, 95:55-61.

38. Lin P, Guo YN, Shi L, Li XJ, Yang H, He Y, Li Q, Dang YW, Wei KL, Chen G: Development of a prognostic index based on an immunogenomic landscape analysis of papillary thyroid cancer. Aging (Albany NY)2019, 11(2):480-500.

\section{Tables}

Table 1 


\begin{tabular}{|llllll|}
\hline semaphorins & CANCER TYPE & FOLD CHANGE & P-VALUE & T-TEST & REFERENCES PMID \\
\hline SEMA3A & GBM & 2.553 & $7.81 \mathrm{E}-5$ & 4.168 & 16697959 \\
\hline SEMA3B & GBM & 13.262 & $5.89 \mathrm{E}-5$ & 8.947 & 15827123 \\
\hline SEMA3E & GBM & 12.146 & 0.014 & 4.338 & 16697959 \\
\hline SEMA3F & GBM & 3.106 & $3.79 \mathrm{E}-05$ & 9.604 & 16697959 \\
\hline SEMA3G & GBM & -2.470 & $1.00 \mathrm{E}-03$ & -3.890 & TCGA \\
\hline SEMA4A & GBM & -2.364 & $2.00 \mathrm{E}-03$ & -5.072 & 16204036 \\
\hline SEMA4D & GBM & -7.051 & $1.00 \mathrm{E}-03$ & -6.124 & TCGA \\
\hline SEMA4F & GBM & -5.566 & 0.005 & -5.394 & 18565887 \\
\hline & GBM & -2.690 & $1.00 \mathrm{E}-03$ & -6.410 & TCGA \\
\hline SEMA5A & GBM & 2.485 & $3.00 \mathrm{E}-03$ & -4.466 & 16204036 \\
\hline SEMA5B & GBM & -2.917 & $3.51 \mathrm{E}-5$ & 7.550 & 18565887 \\
\hline SEMA6A & GBM & 2.439 & 0.004 & 3.528 & TCGA \\
\hline
\end{tabular}

\section{Figures}

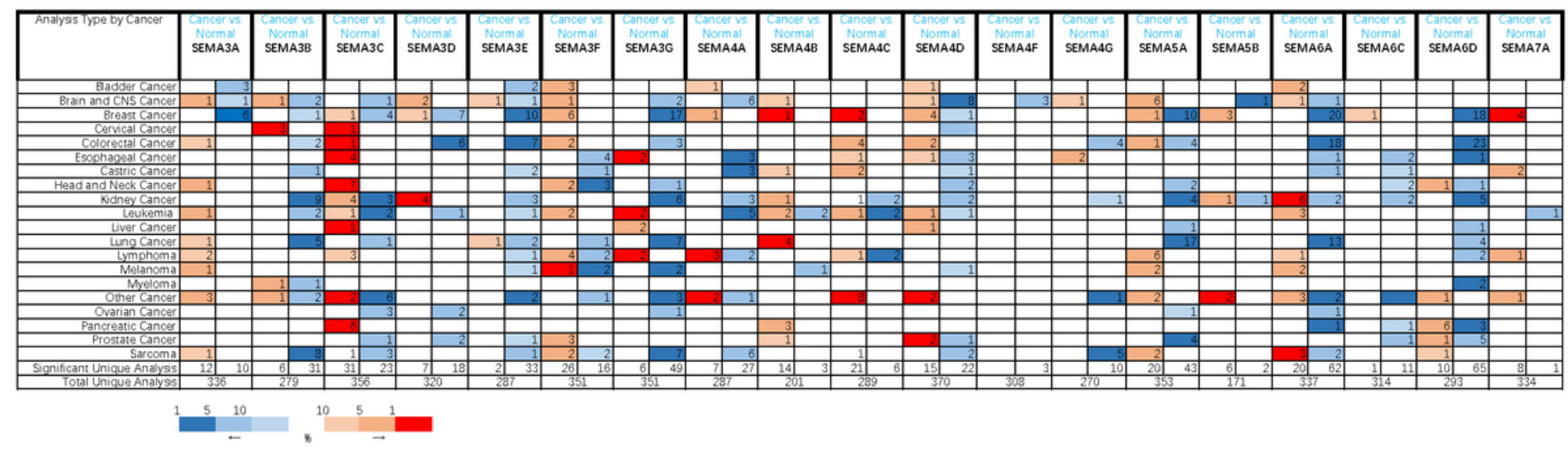

Figure 1

mRNA levels of semaphorins in GBM. The number of datasets is shown with statistically significant mRNA over-expression (red) or down-regulated expression (blue) of semaphorins (ONCOMINE). 
Expression of SEMA3F in GBM based on Sample types

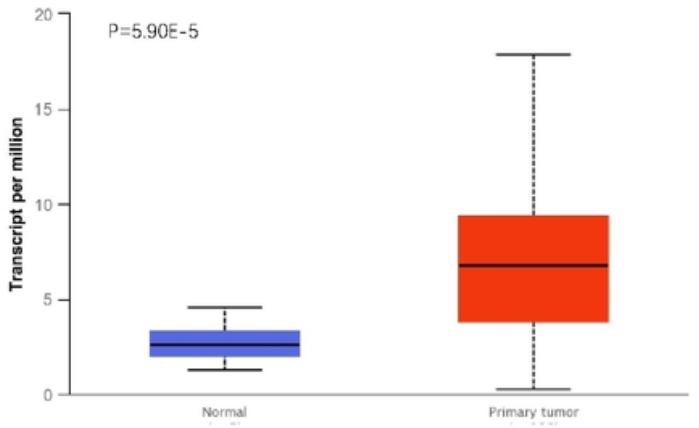

Expression of SEMA4B in GBM based on Sample types

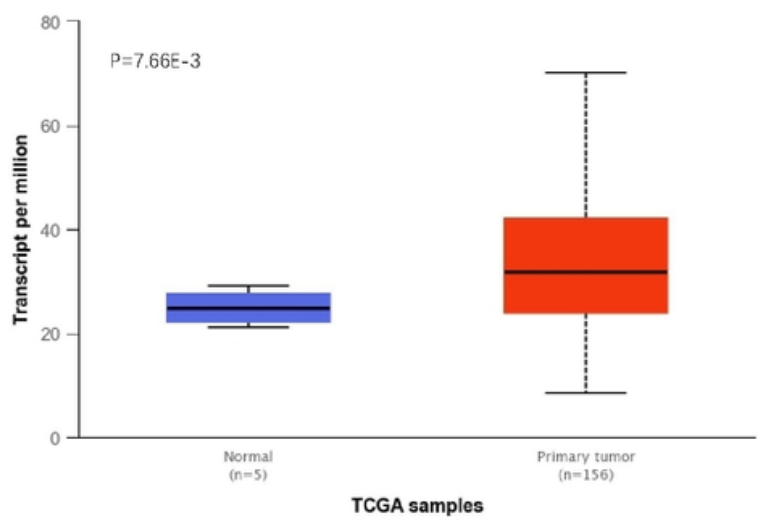

Expression of SEMA4A in GBM based on Sample types

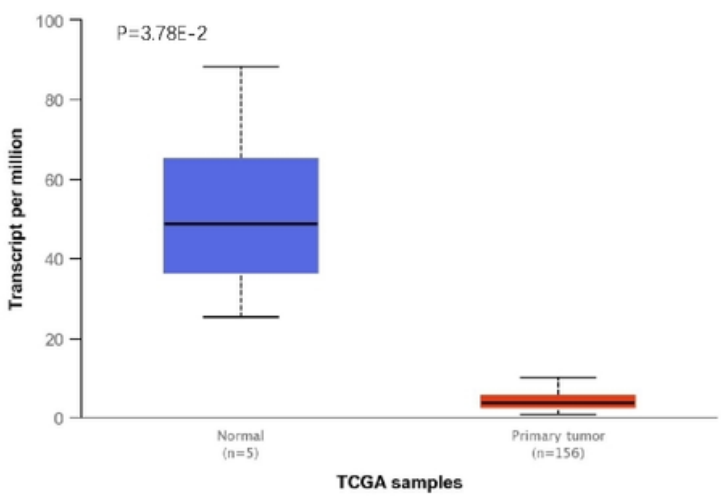

\section{Figure 2}

Transcriptional of semaphorins in GBM (UALCAN). The transcriptional levels of SEMA3A, SEMA3F, SEMA4B, and SEMA4G in GBM tissues were significantly elevated, while SEMA4A was reduced with $p$ value at 0.05 . 


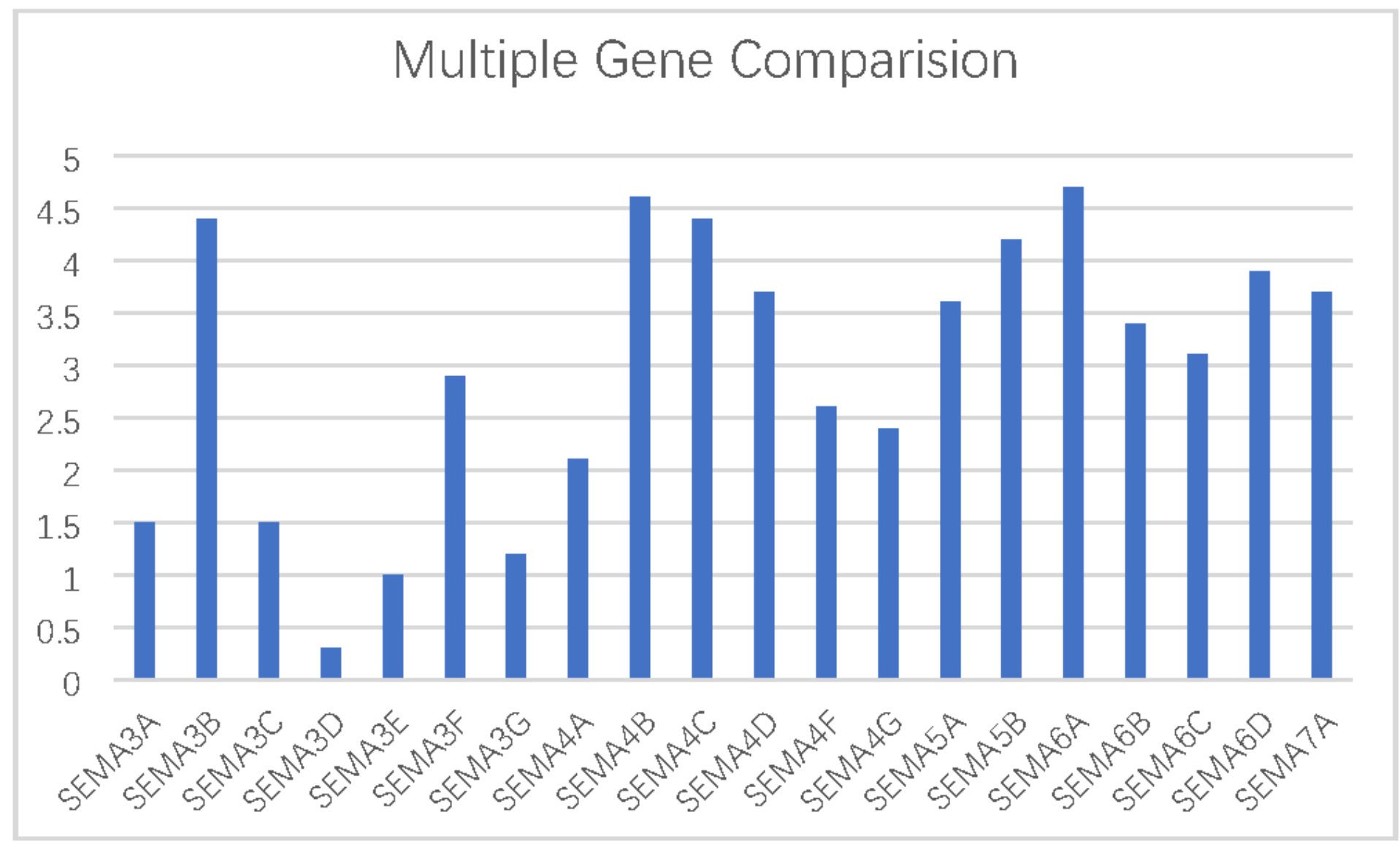

Figure 3

The relative expression of SEMA in GBM (GEPIA). 

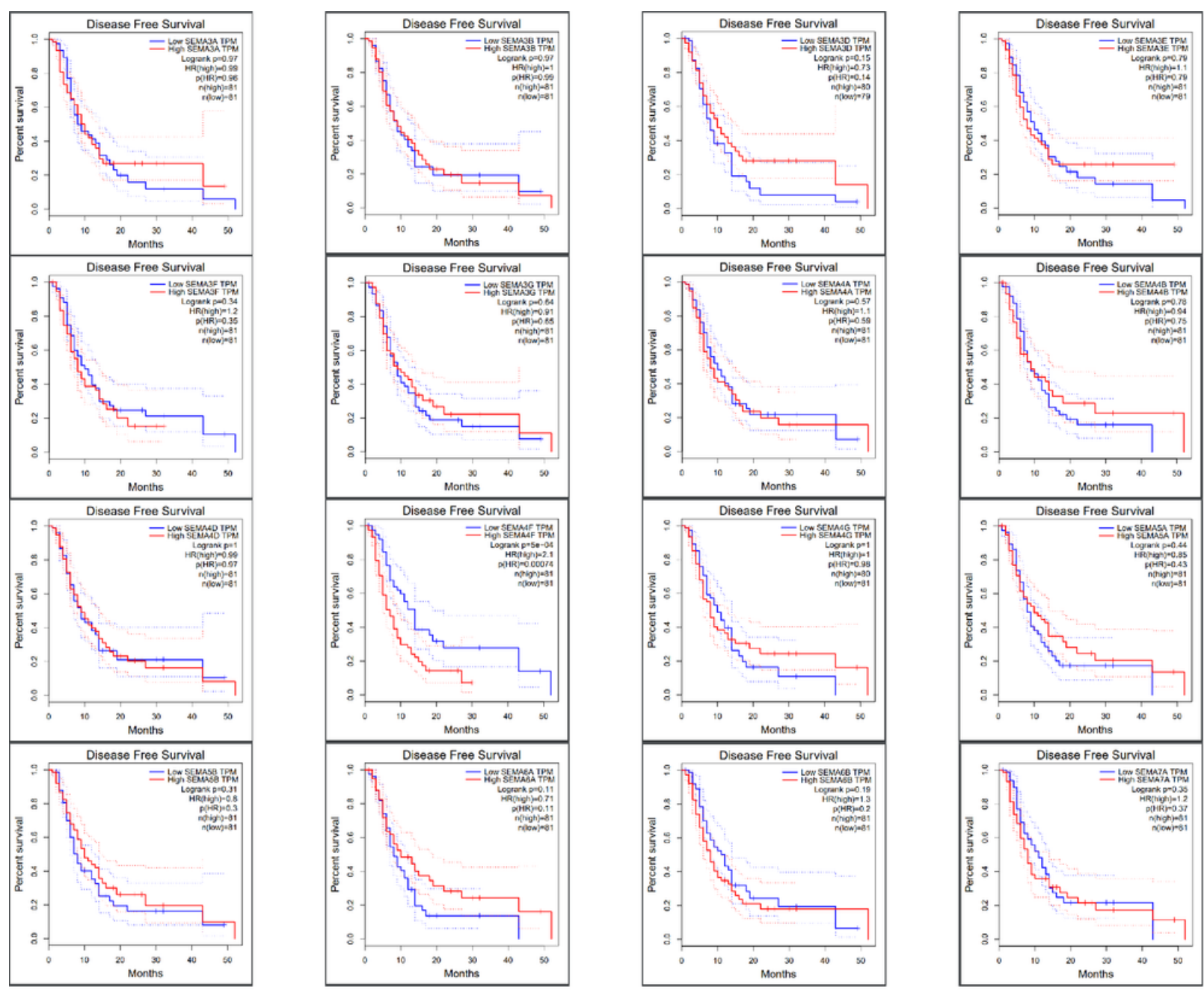

\section{Figure 4}

The value of prognostication of semaphorins in different expressional levels in GBM. And this is the disease-free survival curve (GEPIA). 

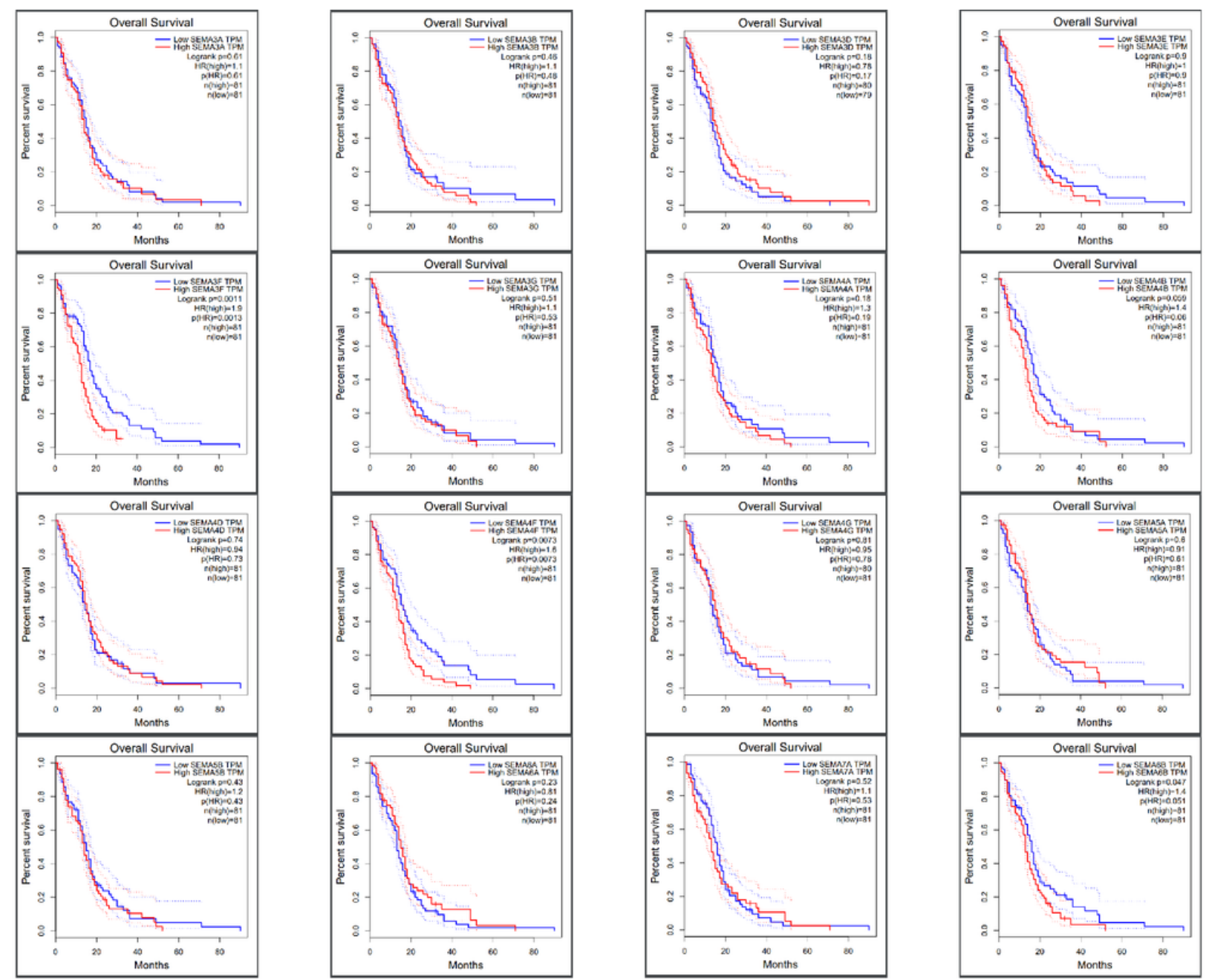

Figure 5

The value of prognostication of semaphorins in GBM patients in the over-all survival curve (GEPIA).

\# Samples per Patient

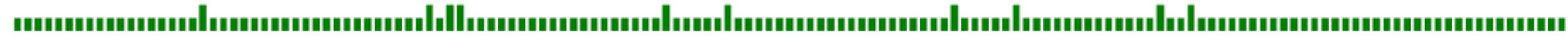

SEMA3A

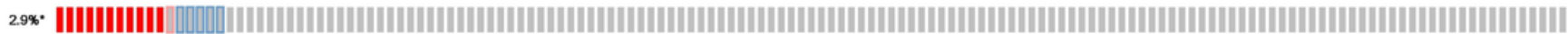

SEMA3B 1.5*

SEMA3D 228 1

SEMA3E 3*·

SEMA3F 24*·

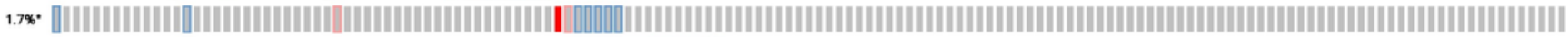
228. 1.2. 228. 22. 1.5* .

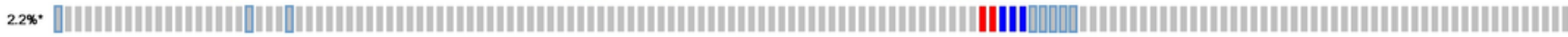
24s. 1.7. 278. 228.

SEMA7A

Genetic Alteration

| Missense Mutation (unknown significance) i| Truncating Mutation (unknown significance)

| Amplification (unknown significance)

| Deep Deletion (unknown significance)

|mPNA High ImRNA Low || No alterations - Not profled 
Genetic alteration. The summary of alterations in differentially expressed semaphorins in GBM.

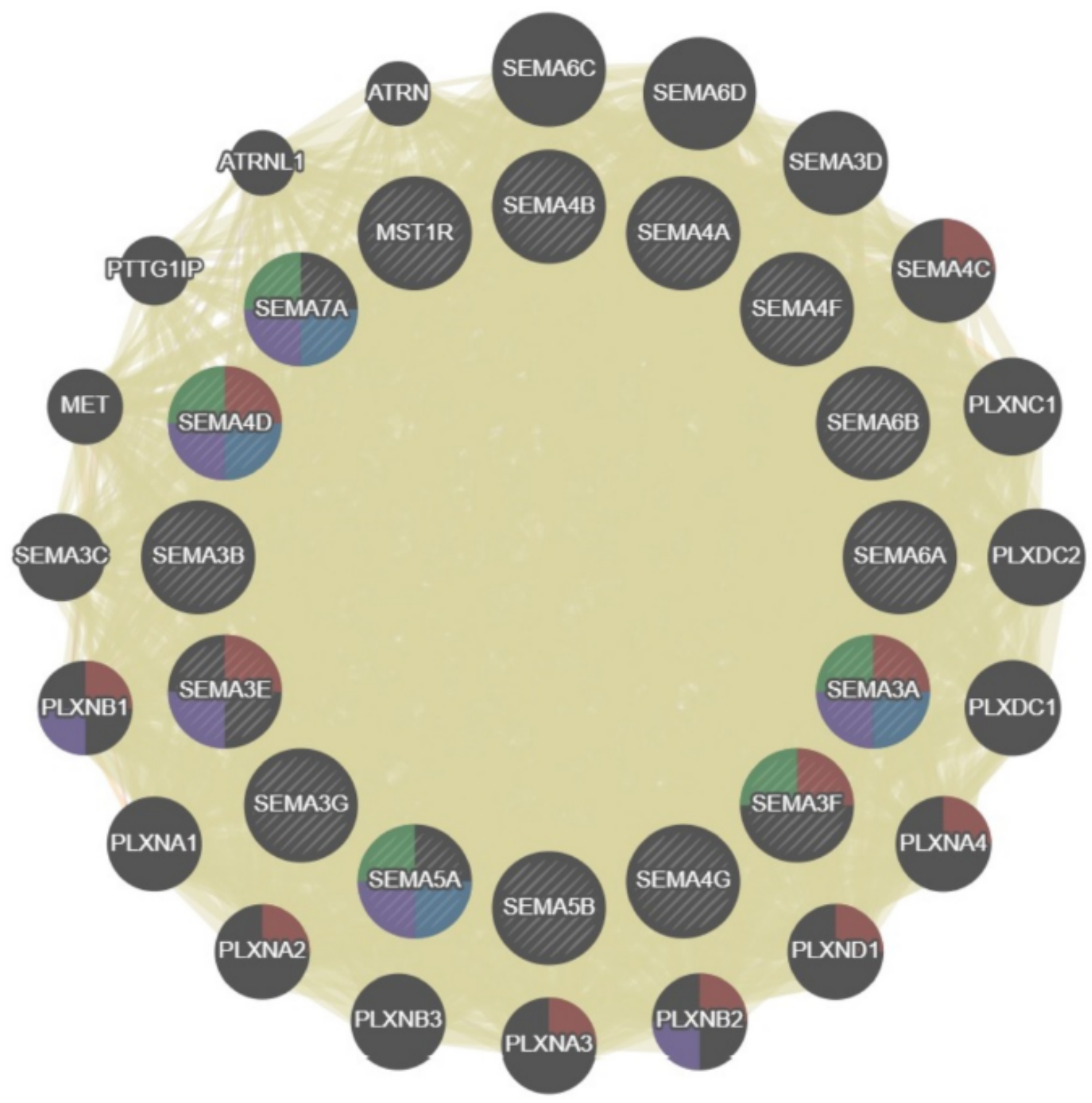

\section{Functions}

- semaphorin-plexin signaling pathway

regulation of extent of cell growth

- regulation of cell morphogenesis

developmental cell growth

\section{Figure 7}

The correlation map of differentially expressed semaphorins in GBM.

\section{Supplementary Files}


This is a list of supplementary files associated with this preprint. Click to download.

- supplementfile.docx 